\title{
Assessment of antitumor activity and acute peripheral neuropathy of I,2-diaminocyclohexane platinum (II)-incorporating micelles (NC-40I6)
}

This article was published in the following Dove Press journal:

International Journal of Nanomedicine

19 June 2014

Number of times this article has been viewed

\author{
Takayoshi Ueno' \\ Kazuhira Endo' \\ Kiyomi Hori ${ }^{2}$ \\ Noriyuki Ozaki² \\ Akira Tsuji \\ Satoru Kondo' \\ Naohiro Wakisaka' \\ Shigeyuki Murono' \\ Kazunori Kataoka ${ }^{3,4}$ \\ Yasuki Kato ${ }^{5}$ \\ Tomokazu Yoshizaki' \\ 'Division of Otolaryngology-Head \\ and Neck Surgery, ${ }^{2}$ Department \\ of Functional Anatomy, Graduate \\ School of Medical Science, Kanazawa \\ University, Kanazawa, Japan; \\ ${ }^{3}$ Department of Materials Engineering, \\ Graduate School of Engineering, \\ ${ }^{4}$ Division of Clinical Biotechnology, \\ Center for Disease Biology and \\ Integrative Medicine, Graduate School \\ of Medicine, The University of Tokyo, \\ Tokyo, Japan; ${ }^{5}$ Research Division, \\ NanoCarrier Co., Ltd, Chiba, Japan
}

Correspondence:Tomokazu Yoshizaki Division of Otolaryngology-Head and Neck Surgery, Graduate School of Medical Science, Kanazawa University, I3-I Takara-machi, Kanazawa, Ishikawa 920-864I, Japan

Tel +8I 762652413

Fax+8I 762344265

Email tomoy@med.kanazawa-u.ac.jp
Abstract: Oxaliplatin, a third-generation platinum compound incorporating oxalate and 1,2-diaminocyclohexane platinum, has been widely used in chemotherapy regimens for the treatment of metastatic colorectal cancer. Because of its wide spectrum of antitumor activity, oxaliplatin has been applied for the treatment of other carcinomas. However, the antitumor activity of single-agent oxaliplatin is insufficient. To increase its antitumor effects, polymeric micellar nanoparticles incorporating 1,2-diaminocyclohexane platinum (NC-4016) have been developed. The present study was designed to evaluate the efficacy of NC-4016 and its association with peripheral neuropathy, which is a primary dose-limiting factor in oxaliplatin therapy. The in vitro antitumor activity of NC-4016 was investigated using human carcinoma cell lines. To investigate the antitumor effects of NC-4016 in vivo, nude mice bearing the human carcinoma cell line $\mathrm{KB}$ were administered $\mathrm{NC}-4016$ or oxaliplatin. The in vitro growth-inhibiting effect of NC-4016 was significantly weaker than that of oxaliplatin. However, the antitumor efficacy of NC-4016 was superior to that of oxaliplatin in vivo. Moreover, we compared the severity of peripheral neuropathy induced by oxaliplatin and NC-4016 in a rat model. Oxaliplatin, NC-4016, or 5\% glucose (control) were administered by a single tail vein injection. In the oxaliplatin-treated rats, neither mechanical nor heat allodynia was observed during the experimental period, whereas cold hyperalgesia/allodynia was observed from day 1 to 7 . Conversely, cold hyperalgesia/allodynia was not observed in the NC-4016-treated rats. The present study demonstrated that the antitumor efficacy of NC-4016 was superior to that of oxaliplatin in a mouse model of human carcinoma cell line KB. In addition, NC-4016-treated rats did not develop acute cold hypersensitivity, which is frequently experienced by patients after oxaliplatin administration.

Keywords: oxaliplatin, carcinoma, chemotherapy, drug delivery system

\section{Introduction}

Oxaliplatin is a third-generation cisplatin derivative in which the chlorine and amine groups have been replaced with oxalate and 1,2-diaminocyclohexane platinum (DACH-Pt), respectively. Oxaliplatin has been widely used as a chemotherapeutic agent for the treatment of metastatic colorectal cancer. ${ }^{1} \mathrm{DACH}-\mathrm{Pt}$ possesses a wide spectrum of activity and is active against cisplatin (CDDP)-resistant tumors. ${ }^{2,3}$ Therefore, oxaliplatin has been used for the treatment of other carcinomas, including gastric, pancreatic, ovarian, breast, and lung carcinomas, in many clinical trials. A Phase II study of single-agent oxaliplatin in 44 advanced head and neck squamous cell carcinoma (HNSCC) patients showed an excellent side effect profile; however, the response rate was only $10 \% .{ }^{4}$ On the other hand, the use of oxaliplatin combined with folinic acid and 5-fluorouracil in 36 advanced HNSCC patients showed a $61 \%$ response rate. ${ }^{5}$ 
These results showed that the antitumor activity of single-agent oxaliplatin is insufficient. With respect to side effects, the incidence of nephrotoxicity and hematological toxicity with oxaliplatin was reportedly lower than that with other platinum $(\mathrm{Pt})$-based chemotherapeutic agents. ${ }^{6}$ However, severe peripheral neuropathy is induced by oxaliplatin therapy and often becomes a dose-limiting factor. ${ }^{7,8}$

Recently, several types of therapeutic nanoparticle platforms, including liposomes, nanoparticles, and polymeric micelles, have been developed on the basis of the idea that these drug delivery systems can facilitate the selective accumulation of chemotherapeutic drugs within the tumor, with decreased distribution to normal tissues and minimal undesirable side effects. ${ }^{9-11}$ Nishiyama and Kataoka, and Nishiyama et al developed several antitumor drugincorporating polymeric micellar nanoparticles, including NC-6004 and NC-4016. ${ }^{12,13}$ NC-6004 is a CDDP-incorporating polymeric micellar nanoparticle. Previously, we demonstrated that NC-6004 had more potential advantages, including greater antitumor activity, improved lymphatic drug delivery, and decreased nephrotoxicity, with regard to head and neck cancer treatment compared with CDDP alone. ${ }^{14} \mathrm{NC}-4016$ is a polymeric micellar nanoparticle developed by Cabral et $\mathrm{al}^{13}$ that incorporates DACH-Pt within the core. The solubility of DACH-Pt in water is much lower than that of CDDP $(0.25 \mathrm{mg} / \mathrm{mL}$ versus $1.2 \mathrm{mg} / \mathrm{mL}){ }^{15}$ therefore, DACH-Pt needs oxalate to enhance its solubility in water. By contrast, the NC-4016 micelles were prepared through polymer-metal complex formation between DACH-Pt and poly(ethylene glycol)-poly(glutamic acid) block copolymers (PEG-P(Glu)) in distilled water after converting DACH-Pt to its aqueous complex by pretreatment with the silver $\left(2^{+}\right)$ salt $(2: 1)$ silver nitrate $\left(\mathrm{AgNO}^{3}\right)$ to increase its solubility in water. In addition to water solubility, PEG-P(Glu) confers considerable stability and has a diameter of $40 \mathrm{~nm}$, which may be sufficient to achieve extended blood circulation, avoid uptake by the reticuloendothelial system, and overcome the permeation barrier of poorly permeable tumors. This mechanism has been recognized as the "enhanced permeability and retention" effect, in which extravasated high-molecular-weight micelles pass through leaky tumor capillary fenestrations resulting from abnormalities in angiogenesis at the tumor site, thus leading to accumulation and retention of the particles for prolonged durations. ${ }^{13}$

In the present study, we analyzed the antitumor effect of NC-4016 in a mouse model of human carcinoma cell line $\mathrm{KB}$. In addition, we compared NC-4016 with oxaliplatin in a rat model of peripheral neuropathy, which is a primary dose-limiting factor in oxaliplatin therapy.

\section{Materials and methods}

\section{In vitro growth inhibition assay}

Oxaliplatin was obtained from Wako Pure Chemical Industries, Ltd. (Osaka, Japan), while NC-4016 was supplied by NanoCarrier Co., Ltd. (Chiba, Japan). The human oral carcinoma cell lines, KB, OSC-19 (human squamous cell carcinoma cell line from tongue), OSC-20 (human squamous cell carcinoma cell line from tongue metastasized to neck lymph node), HSC-3 (human oral squamous carcinoma cell line with high metastatic potential), and HSC-4 (human squamous carcinoma cell line from tongue) were used in the present study. The KB cell lines were kindly provided by Shin-ichi Akiyama. ${ }^{16} \mathrm{~KB}$ is now known to be a subline of the ubiquitous keratin-forming tumor cell line HeLa (http://www.ncbi.nlm. nih.gov/mesh? $\mathrm{Db}=$ mesh\&term $=\mathrm{KB}+\mathrm{Cells}$ ). The KB, HSC-3, and HSC-4 cells were maintained in Dulbecco's Modified Eagle's Medium supplemented with 10\% fetal bovine serum and $1 \%$ antibacterial agent, whereas the OSC-19 and OSC-20 cells were maintained in Eagle's Minimum Essential Medium supplemented with $10 \%$ fetal bovine serum and $1 \%$ antibacterial agent. The A-3-(4,5-dimethylthiazol2-yl)-5-(3-carboxymethoxyphenyl)-2-(4-sulfophenyl)-2Htetrazolium salt (MTS) assay was performed to assess the effects of cell proliferation, using the CellTiter $96^{\circledR}$ AQueous One Solution Cell Proliferation Assay (Promega Corp, Fitchburg, WI, USA). Briefly, cells were seeded in 96-well culture plates at a density of $2 \times 10^{3}$ cells/well. After a 24 -hour incubation period with either $5 \%$ glucose (control), a graded concentration of oxaliplatin, or NC-4016, the MTS reagents were added to each well and incubated for 48 or 72 hours. Optical density was read with an EMax Endpoint Elisa Microplate Reader (Molecular Devices LLC, Sunnyvale, CA, USA) at a wavelength of $490 \mathrm{~nm}$. The half maximal inhibitory concentration $\left(\mathrm{IC}_{50}\right)$ values represented drug concentrations that decreased the mean absorbance at $490 \mathrm{~nm}$ to $50 \%$ of that in the untreated control well.

\section{Evaluation of antitumor activity}

Six-week-old female BALB/c-nu/nu mice (Charles River Laboratories, Inc., Wilmington, MA, USA) were subcutaneously inoculated with $\mathrm{KB}$ cells $\left(1 \times 10^{5}\right)$. After 14 days, the tumor diameters reached approximately $10 \mathrm{~mm}$, and the tumor-bearing mice were randomly allocated to three drug administration groups: a control ( $5 \%$ glucose solution) group, an oxaliplatin (4 mg/kg body weight [BW], the equivalent of $1.96 \mathrm{mg} / \mathrm{kg} \mathrm{BW}$ of elemental $\mathrm{Pt}$ ) treatment group, and an $\mathrm{NC}-4016$ (the equivalent of $1.96 \mathrm{mg} / \mathrm{kg} \mathrm{BW}$ of Pt) treatment group. The drugs were intravenously injected via the tail vein at 14,21 , and 28 days after tumor transplantation. 
The major and minor axes of tumor were measured weekly. The tumor volume was calculated using the formula:

$$
\text { tumor volume }\left(\mathrm{mm}^{3}\right)=1 / 2 \times \mathrm{L} \times \mathrm{W}^{2} \text {, }
$$

where $\mathrm{L}$ is the major axis and $\mathrm{W}$ is the minor axis. ${ }^{17,18}$ The mice were sacrificed on posttransplantation day 35 , and the tumors were excised and weighed.

\section{Plasma platinum concentration and tumor platinum accumulation}

In the same manner as described above, 6-week-old female $\mathrm{BALB} / \mathrm{c}-\mathrm{nu} / \mathrm{nu}$ mice were subcutaneously inoculated with KB cells. After 14 days, a single dose of either oxaliplatin or NC-4016 was intravenously administered at a dose of $1.96 \mathrm{mg} / \mathrm{kg}$ elemental Pt. Then, the mice were sacrificed at defined time periods (1, 24, 48, and 168 hours), and the tumors were excised. Blood samples were collected from the inferior vena cava and heparinized and centrifuged to obtain plasma. The plasma and tumor Pt concentrations were measured using atomic absorption spectrometry (NAC Co., Ltd., Tokyo, Japan).

\section{Assessment of drug-induced peripheral neuropathy}

\section{Experimental animals}

Young adult male Sprague-Dawley rats (BW 200-250 g) were purchased from Charles River Laboratories, Inc.) and housed in a temperature-controlled room $\left(23^{\circ} \mathrm{C}\right)$ under a 12-hour light-dark cycle, with ad libitum access to food and water.

\section{Ethics statement}

This study was conducted under the auspices of the Local Animal Ethics Committee in accordance with the Regulations for Animal Experiments in the Kanazawa University Graduate School of Medicine (permit numbers: 122433 and 122465), the Act on Welfare and Management of Animals adopted by the Government of Japan (http://www.cas.go.jp/jp/ seisaku/hourei/data/AWMA.pdf), and the guidelines of the International Association for the Study of Pain (http://www. iasp-pain.org/). All surgery was performed under sodium pentobarbital anesthesia, and all efforts were made to minimize animal suffering.

\section{Oxaliplatin and NC-40I6 administration}

The rats were divided into three treatment groups (oxaliplatin; NC-4016; and 5\% glucose, as a control). Oxaliplatin or NC-4016 was dissolved in 5\% glucose solution and administered to the respective rats via a single intravenous injection at a dose of $4 \mathrm{mg} / \mathrm{kg} \mathrm{BW}(4.0 \mathrm{~mL} / \mathrm{kg}$ volume, the equivalent of $1.96 \mathrm{mg} / \mathrm{kg} \mathrm{BW}$ of $\mathrm{Pt}$ ) to the tail vein. The control group was injected with the same volume of 5\% glucose solution, calculated according to the BW of each $\operatorname{animal}(4 \mathrm{~mL} / \mathrm{kg})$.

\section{Behavioral testing}

Behavioral analyses were conducted by experimenters blinded to the experimental conditions.

\section{Acetone test}

The acetone test was performed before and on days 1 , 2, 3, 5, 7, 9, and 14 after drug administration. Briefly, each animal was placed in a transparent plastic box with a wire mesh floor, and a drop $(0.05 \mathrm{~mL})$ of acetone was applied to the center of the plantar surface of the hind paw. The response of the rat was monitored for the following 40 seconds and graded on a four-point scale defined by Flatters and Bennett. This was scored as: ${ }^{19} 0=$ no response; $1=$ quick withdrawal, flick, or stamp of the paw; $2=$ prolonged withdrawal or repeated flicking of the paw; and $3=$ repeated flicking of the paw with licking directed at the plantar surface of the paw. Acetone was applied alternately to each hind paw for a total of three times, and the responses were scored. Cumulative scores were then generated by adding the six scores for each rat, yielding a minimum score of 0 and a maximum score of 18 .

\section{Assessment of cold and heat allodynia}

Conscious rats were gently wrapped with a string cover, and thermal stimulation was applied using a Peltier probe controller (UDH-300; Unique Medical Co., Ltd, Tokyo, Japan). The tip of the probe $(5 \times 5 \mathrm{~mm})$ was applied to the plantar surface of the hind paw. Then, the temperature was serially increased from $30^{\circ} \mathrm{C}$ to $55^{\circ} \mathrm{C}\left(+1.0^{\circ} \mathrm{C} / \mathrm{s}\right)$ or decreased from $30^{\circ} \mathrm{C}$ to $5^{\circ} \mathrm{C}\left(-1.0^{\circ} \mathrm{C} / \mathrm{s}\right)$. Before measurement, the probe, at $30^{\circ} \mathrm{C}$, was applied to the hind paw for at least 60 seconds. The temperature that induced paw withdrawal was measured as a nociceptive thermal threshold. Each animal was tested 10 times, at 5-minute intervals.

\section{Assessment of mechanical allodynia}

The von Frey test was performed before and on days 1, 3, 6, 10, and 13 after drug administration. Briefly, each rat was placed in a cage with an elevated metal floor covered with transparent plastic boxes and allowed to acclimatize to the novel surroundings for at least 20 minutes prior to the 
initiation of behavioral testing. Then, the paw withdrawal threshold value was measured using a von Frey-type filament (diameter $0.5 \mathrm{~mm}$ ), by applying increasing force to the plantar surface of the hind paw, starting below the detection threshold and increasing until the animal withdrew its paw (Dynamic Plantar Aesthesiometer; Ugo Basile Srl, Comerio, Italy). The force was registered automatically, in grams, when the paw was withdrawn. A $50 \mathrm{~g}$ limit was imposed as the cutoff to preclude tissue damage. Each animal was tested five times at 5-minute intervals, and the five measurements were averaged.

\section{Statistical analysis}

All data are expressed as means \pm standard error of the mean. The values were analyzed using two-way repeated-measures analysis of variance (ANOVA) followed by the Bonferroni post hoc test, to determine differences among groups. SPSS statistical software (Version 18; IBM, Armonk, NY, USA) was used for all analyses. A $P$-value of $<0.05$ was considered statistically significant.

\section{Results}

\section{Sensitivity of human carcinoma cell lines to oxaliplatin and NC-40I6}

The cytotoxicity of NC-4016 was compared with that of oxaliplatin, in human carcinoma cell lines: $\mathrm{IC}_{50}$ values were calculated from dose-survival curves obtained from MTS assays performed 48 and 72 hours after treatment. A graphical representation of the MTS assay of the KB cells is presented (Figure 1). The $\mathrm{IC}_{50}$ values for oxaliplatin were 15.2-78.4 $\mu \mathrm{M}$ at 48 hours and 5.2-69.2 $\mu \mathrm{M}$ at 72 hours. NC-4016 showed a lower range of growth inhibition compared with oxaliplatin, with $\mathrm{IC}_{50}$ values of $63.8-771.2 \mu \mathrm{M}$ at 48 hours and $13.6-334.2 \mu \mathrm{M}$ at 72 hours. The cytotoxicity of oxaliplatin in vitro was 1.2- to 9.8-fold greater than that of NC-4016.

\section{Antitumor activity in KB xenografts}

The antitumor activity of oxaliplatin and NC-4016 was a evaluated in mice with oral carcinoma. All mice in each group received a single intravenous injection of the respective drug or glucose, via the tail vein, on days 14, 21, and 28 after transplantation. All mice survived the experiment. All treatments did not reduce body weight.

In the oxaliplatin and $\mathrm{NC}-4016$ treatment groups, the tumor volume was significantly decreased compared with that in the control group, from 1 week after initial drug administration in the $\mathrm{NC}-4016$ treatment group and from 2 weeks after initial drug administration in the

\begin{tabular}{llllll}
\hline Cell line & \multicolumn{3}{l}{$\mathrm{IC}_{50}(\mu \mathrm{M})$} & & \\
\cline { 2 - 3 } & $\mathbf{4 8}$ hours & & & $\mathbf{7 2}$ hours & \\
\cline { 2 - 3 } \cline { 5 - 6 } & Oxaliplatin & NC-4016 & & Oxaliplatin & NC-4016 \\
\hline KB & 30.5 & 85.6 & & 14.2 & 30.2 \\
OSC-19 & 78.4 & 771.2 & & 69.2 & 334.3 \\
OSC-20 & 60.2 & 510.2 & & 55.3 & 276.5 \\
HSC-3 & 15.2 & 90.6 & & 5.2 & 13.6 \\
HSC-4 & 18.6 & 63.8 & & 17.6 & 21.6 \\
\hline
\end{tabular}

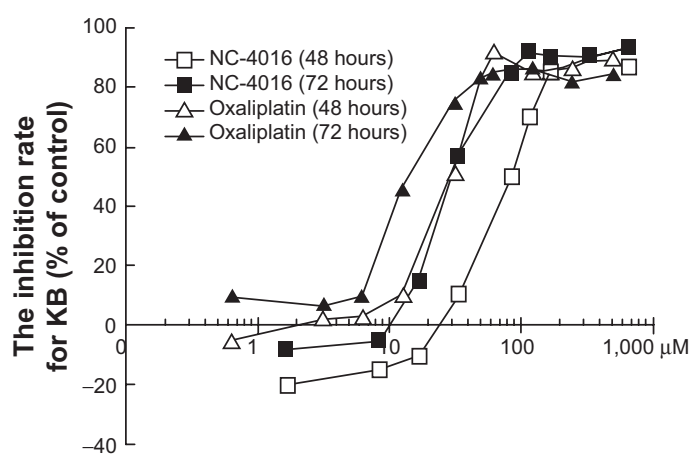

Figure I The $50 \%$ inhibitory concentration $\left(\mathrm{IC}_{50}\right)$ values of oxaliplatin and NC-40I6.

oxaliplatin treatment group (Figure 2A). On day 35, tumor growth was significantly lower in the NC-4016 group than in the control group (Figure 2B) $(-74 \%[1.41 \pm 0.44 \mathrm{~g}$ versus $3.82 \pm 1.05 \mathrm{~g}])(P=0.000)$. Similarly, tumor growth in the oxaliplatin group was also significantly lower than that in the control group (Figure 2B) $(-33 \%[2.54 \pm 1.05 \mathrm{~g}$ versus $3.82 \pm 1.05 \mathrm{~g}])(P=0.003)$. In addition, there was a significant difference in tumor growth inhibition between the NC-4016 and oxaliplatin groups (Figure 2B) $(P=0.009)$.

\section{Platinum concentration in the plasma and tumor after NC-40I6 treatment}

The time course of elemental Pt concentration in the plasma and tumor after intravenous administration of either oxaliplatin or NC-4016 was measured (Figure 3). Plasma concentration and tumor accumulation of $\mathrm{Pt}$ after administration of NC-4016 peaked at 1 hour and 48 hours, respectively. Pt was rapidly cleared from circulation in the oxaliplatin group. Areas under the curve at 0-168 hours for plasma and tumor Pt concentrations in the NC-4016 group were significantly higher than those in the oxaliplatin group. However, on day 7 (168 hours), plasma and tumor Pt concentrations were undetectable in both groups.

\section{Behavioral testing}

\section{Cold hyperalgesia/allodynia}

In the acetone test, oxaliplatin significantly increased the cold sensitivity score compared with that in the control group on 

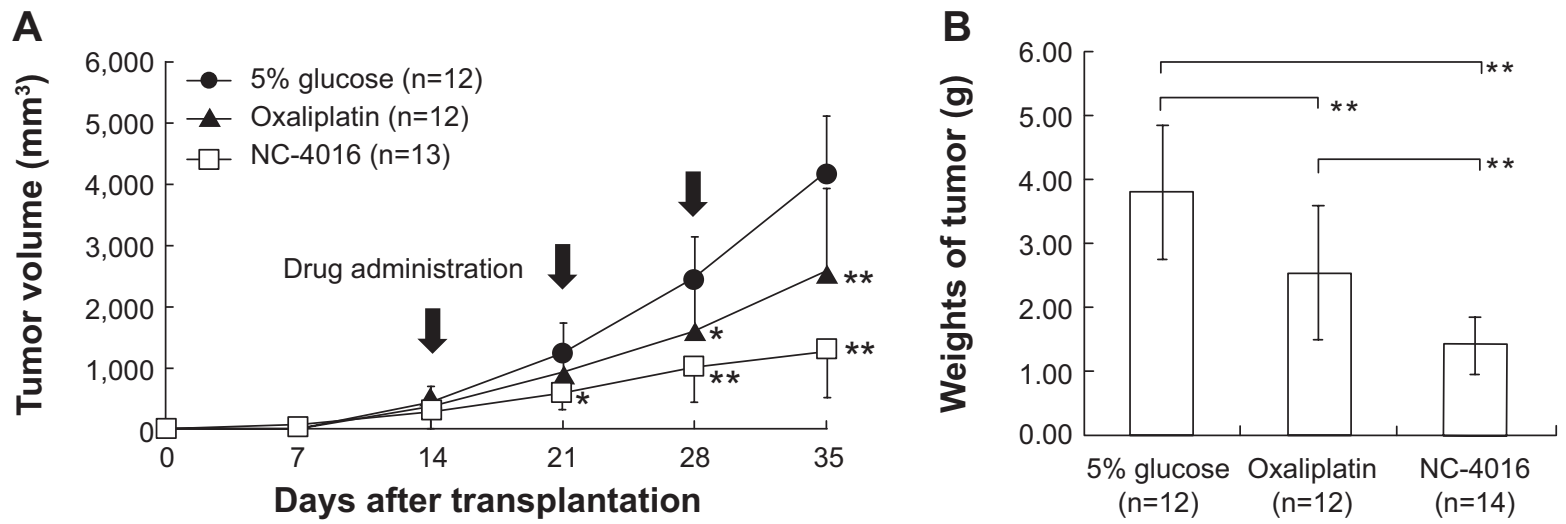

Figure 2 Effects of oxaliplatin or NC-4016 in KB mouse models.

Notes: Each data point is the mean value ( \pm SEM) of the largest tumor diameter $(\mathbf{A})$. On day 35, the tumor was excised and weighed (B). Data were analyzed using a one-way (A) or two-way repeated-measures (B) ANOVA, followed by the Bonferroni post hoc test. $* P<0.05$, ${ }^{*} * P<0.01$ compared with $5 \%$ glucose (control).

Abbreviations: ANOVA, analysis of variance; SEM, standard error of the mean.

days 1, 2, 3, 5, and 7 after single drug injection (Figure 4A), whereas NC-4016 did not. Similarly, the oxaliplatin-treated rats exhibited a significantly higher threshold temperature for withdrawal from cold stimuli compared with the control rats on day 1 through at least day 5 (Figure 4B), whereas the NC-4016-treated rats did not.

\section{Heat sensitivity}

Heat sensitivity in the hind paws was evaluated following single drug injection. No change in the withdrawal threshold was observed during the 2 weeks in all groups (Figure 4C).

\section{Mechanical sensitivity}

Mechanical sensitivity of the hind paws was evaluated using the von Frey test following single drug injection. No change in the withdrawal threshold was observed during the 2 weeks in all groups (Figure 4D).

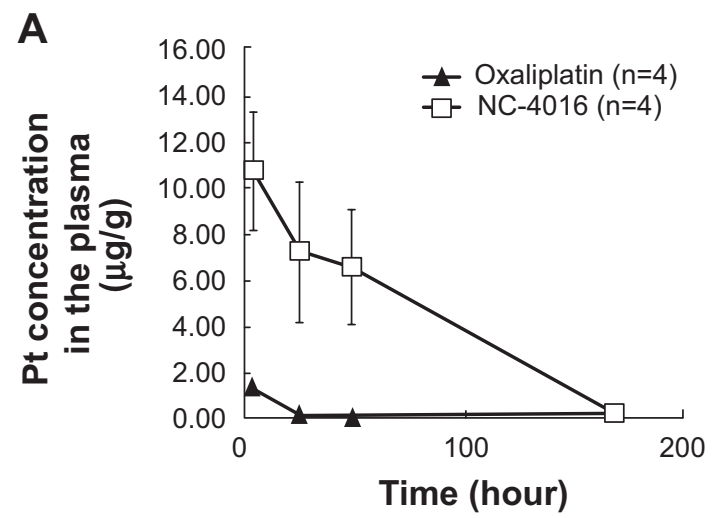

\section{Discussion}

The in vitro growth-inhibiting effect of the DACH-Pt-loaded micelle NC-4016 was significantly weaker than that of oxaliplatin, probably because of the slow-release behavior of DACH-Pt complexes from the micelles. The slow-release profile of Pt complexes from the DACH-Pt-loaded micelles was presented in the previous physicochemical study. ${ }^{13,20} \mathrm{In}$ addition to the in vitro assay, we evaluated the in vivo antitumor activity of oxaliplatin and NC-4016 in a mouse model of human carcinoma. Decreases in tumor weights after the administration of NC-4016 and oxaliplatin were approximately $74 \%$ and $33 \%$, respectively, with a significant difference between them. The findings suggest that the antitumor effect of NC-4016 against human carcinoma cell lines is more potent than that of oxaliplatin in vivo, but not in vitro.

In the present study, NC-4016 resulted in high and extended Pt concentrations within the tumors. Pt

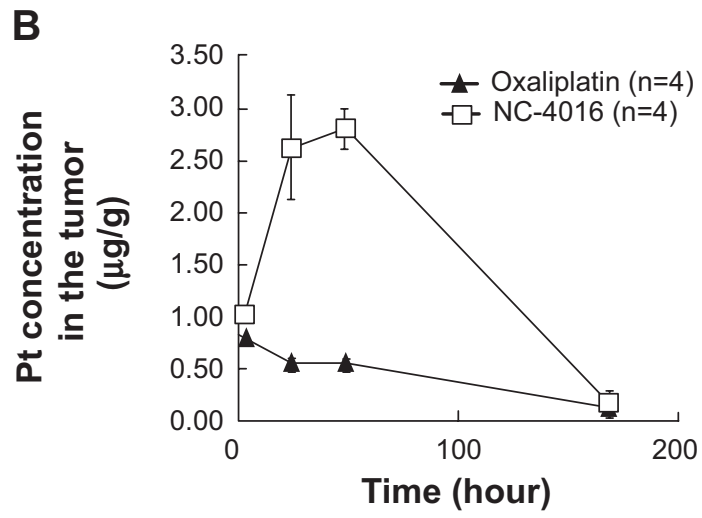

Figure 3 The time course of elemental platinum concentrations in the plasma (A) and tumor (B), after intravenous administration of oxaliplatin (4 mg/kg) or NC-40I6 (an equivalent dose to $4 \mathrm{mg} / \mathrm{kg}$ ).

Notes: Each drug was administered to KB-bearing nude mice (female, $\mathrm{n}=4$ ). Data are presented as mean $\pm \mathrm{SEM}$.

Abbreviation: SEM, standard error of the mean. 
A
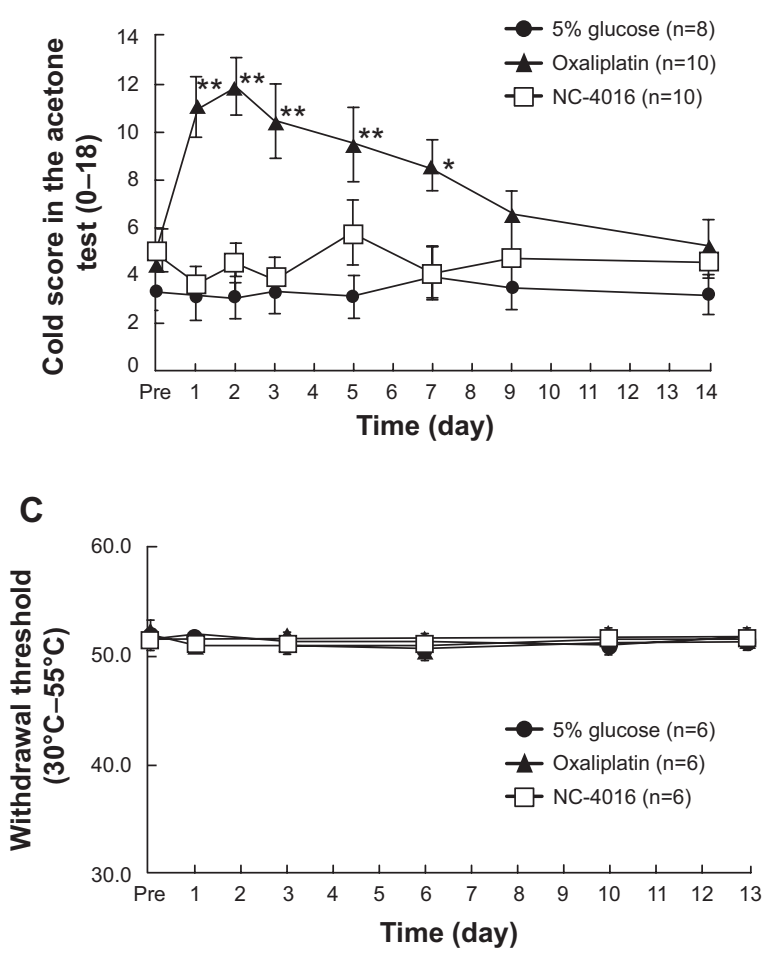

B

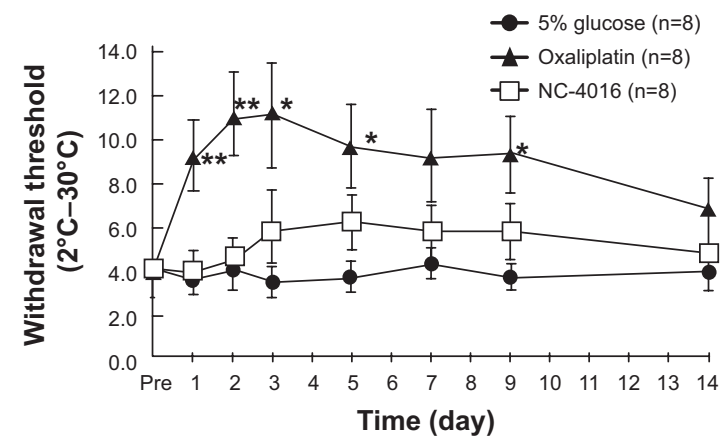

D

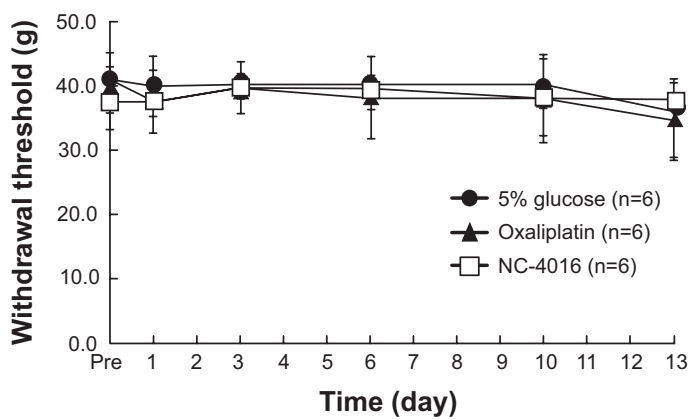

Figure 4 Changes in cold sensitivity score (A), cold threshold temperature (B), heat threshold temperature (C), and mechanical threshold (D), following administration of either oxaliplatin, NC-40I6, or $5 \%$ glucose (control).

Notes: Data are presented as mean \pm SEM. Data were analyzed using a two-way repeated-measures ANOVA, followed by the Bonferroni post hoc test. $* P<0.05$, * $* * 0.01$ compared with $5 \%$ glucose (control).

Abbreviations: ANOVA, analysis of variance; SEM, standard error of the mean.

concentration in the tumor decreased continuously with oxaliplatin, while it decreased after initial increase with NC-4016. This is worthy of note. In addition, the previous study showed that NC-4016 preferentially accumulates within the tumor tissue compared with other tissue. ${ }^{20,21}$ Higher Pt concentrations in the tumor may indicate a stronger antitumor effect of NC-4016 compared with that of oxaliplatin in vivo.

Oxaliplatin lacks the nephrotoxicity associated with cisplatin, and the severe myelosuppression associated with carboplatin; ${ }^{22}$ however, it may induce severe peripheral neuropathy. Severe oxaliplatin-induced peripheral neuropathy is characterized by two types of neurological symptoms. The first, acute neuropathy, occurs within hours after infusion in $90 \%$ of patients and includes acral paresthesia and dysesthesia triggered or enhanced by exposure to cold. This type of neuropathy has not been observed before with other Pt-based agents; therefore, it may be a specific effect of oxaliplatin.

The other is chronic neuropathy, which is characterized by the loss of sensory and motor function after long-term oxaliplatin treatment. Chronic neuropathy is similar to cisplatin-induced neurological symptoms. ${ }^{23}$
A rat and mouse model of oxaliplatin-induced neuropathic pain was developed by Ling et al through repetitive intravenous injections of oxaliplatin. ${ }^{24}$ Assessment of cold and heat allodynia with the Peltier probe controller (UDH-300) was impossible in a mouse model. Therefore, we assessed a rat model of oxaliplatin-induced neuropathic pain in the present study. Acute cold hypersensitivity was not produced by other Pt-based chemotherapeutic agents, which are known to induce chronic peripheral neuropathy following repeated administration. Therefore, oxaliplatin, NC-4016, or $5 \%$ glucose (control) were administered by a single intravenous injection, via the tail vein, to assess pure acute peripheral neuropathy. In rats treated with oxaliplatin, neither mechanical nor heat allodynia was observed during the behavioral experiments, whereas cold hyperalgesia/allodynia was observed from days 1 to 7 . In the previous study, repeated administration of oxaliplatin caused cold hyperalgesia in the early phase and mechanical allodynia in the late phase (2 weeks after first administration). ${ }^{25-27}$ Conversely, cold hyperalgesia/allodynia was not observed in the NC-4016treated rats. Oxalate is well known as a chelator of both $\mathrm{Ca}_{2}{ }^{+}$ and $\mathrm{Mg}^{+}$. In a recent clinical study, $\mathrm{Ca}_{2}{ }^{+}$and $\mathrm{Mg}^{+}$infusions 
were shown to decrease oxaliplatin-induced neuropathy without affecting anticancer efficacy. ${ }^{28}$ Furthermore, recent evidence using an in vitro electrophysiological method showed that oxalate blocked voltage-gated $\mathrm{Na}^{+}$channels. ${ }^{29}$ Therefore, the lack of oxalate in NC-4016 may contribute to the decreased cold hyperalgesia/allodynia. The slow-release behavior of DACH-Pt complexes from the micelles may also decrease the incidence of acute neuropathy.

In the present study, we did not examine the effects of repeated injections of oxaliplatin and NC-4016. Therefore, our model is insufficient to assess chronic peripheral neuropathy. However, with repeated administration, acute symptoms may increase in both duration and severity, and patients may experience symptoms of acute and chronic neuropathy. Although the symptoms associated with acute peripheral sensory and motor nerve hyperexcitability seem to be transient in nature, those of chronic neuropathy can be distressing.

In conclusion, the present study demonstrated that the antitumor efficacy of NC-4016 was superior to that of oxaliplatin in a mouse model of human carcinoma cell line KB. In addition, the incidence of acute neuropathy symptoms, which has been frequently experienced by patients after oxaliplatin administration, was lower with NC-4016 than with oxaliplatin, in the rat model. Currently, the efficacy of NC-4016 is being assessed in a Phase I clinical trial.

\section{Acknowledgments}

The KB cells were provided by Shin-ichi Akiyama, Shigeru Oiso, and Tatsuhiko Furukawa. This work was supported by the Japan Society for the Promotion of Science KAKENHI (grant numbers 22791579 and 2365972) and a Grant for Clinical Cancer Research from the Ministry of Health, Labour and Welfare of Japan.

\section{Disclosure}

The authors report no conflicts of interest in this work.

\section{References}

1. Kidani Y, Noji M, Tashiro T. Antitumor activity of platinum (II) complexes of 1,2-diamino-cyclohexane isomers. Gann. 1980;71(5):637-643.

2. Rixe O, Ortuzar W, Alvarez M, et al. Oxaliplatin, tetraplatin, cisplatin, and carboplatin: spectrum of activity in drug-resistant cell lines and in the cell lines of the National Cancer Institute's Anticancer Drug Screen panel. Biochem Pharmacol. 1996;52(12):1855-1865.

3. Tashiro T, Kawada Y, Sakurai Y, Kidani Y. Antitumor activity of a new platinum complex, oxalato (trans-1-1,2-diaminocyclohexane) platinum (II): new experimental data. Biomed Pharmacother. 1989; 43(4):251-260.

4. Degardin M, Cappelaere P, Krakowski I, Fargeot P, Cupissol D, Brienza S. Phase II trial of oxaliplatin (L-OHP) in advanced, recurrent and/or metastatic squamous cell carcinoma of the head and neck. Eur $J$ Cancer B Oral Oncol. 1996;32B(4):278-279.
5. Raguse JD, Gath HJ, Oettle H, Bier J. Oxaliplatin, folinic acid and 5 -fluorouracil (OFF) in patients with recurrent advanced head and neck cancer: a phase II feasibility study. Oral Oncol. 2006;42(6):614-618.

6. Desoize B, Madoulet C. Particular aspects of platinum compounds used at present in cancer treatment. Crit Rev Oncol Hematol. 2002; 42(3):317-325.

7. Gamelin E, Gamelin L, Bossi L, Quasthoff S. Clinical aspects and molecular basis of oxaliplatin neurotoxicity: current management and development of preventive measures. Semin Oncol. 2002;29 (5 Suppl 15):21-33.

8. Grothey A. Oxaliplatin-safety profile: neurotoxicity. Semin Oncol. 2003; 30(4 Suppl 15):5-13.

9. Davis ME, Chen ZG, Shin DM. Nanoparticle therapeutics: an emerging treatment modality for cancer. Nat Rev Drug Discov. 2008;7(9):771-782.

10. Torchilin VP. Recent advances with liposomes as pharmaceutical carriers. Nat Rev Drug Discov. 2005;4(2):145-160.

11. Nishiyama N, Kataoka K. Current state, achievements, and future prospects of polymeric micelles as nanocarriers for drug and gene delivery. Pharmacol Ther. 2006;112(3):630-648.

12. Nishiyama N, Okazaki S, Cabral H, et al. Novel cisplatin-incorporated polymeric micelles can eradicate solid tumors in mice. Cancer Res. 2003;63(24):8977-8983.

13. Cabral H, Nishiyama N, Okazaki S, Koyama H, Kataoka K. Preparation and biological properties of dichloro(1,2-diaminocyclohexane) platinum (II) (DACHPt)-loaded polymeric micelles. J Control Release. 2005; 101(1-3):223-232.

14. Endo K, Ueno T, Kondo S, et al. Tumor-targeted chemotherapy with the nanopolymer-based drug NC-6004 for oral squamous cell carcinoma. Cancer Sci. 2013;104(3):369-374.

15. Kidani Y, Inagaki K, Iigo M, Hoshi A, Kuretani K. Antitumor activity of 1,2-diaminocyclohexane-platinum complexes against sarcoma-180 ascites form. J Med Chem. 1978;21(12):1315-1318.

16. Fujii R, Mutoh M, Niwa K, et al. Active efflux system for cisplatin in cisplatin-resistant human KB cells. Jpn J Cancer Res. 1994;85(4): 426-433.

17. Nathan CO, Amirghahari N, Rong X, et al. Mammalian target of rapamycin inhibitors as possible adjuvant therapy for microscopic residual disease in head and neck squamous cell cancer. Cancer Res. 2007;67(5): 2160-2168.

18. Carlsson G, Gullberg B, Hafström L. Estimation of liver tumor volume using different formulas - an experimental study in rats. $J$ Cancer Res Clin Oncol. 1983;105(1):20-23.

19. Flatters SJ, Bennett GJ. Ethosuximide reverses paclitaxel- and vincristine-induced painful peripheral neuropathy. Pain. 2004;109(1-2): $150-161$.

20. Cabral H, Nishiyama N, Kataoka K. Optimization of (1,2-diaminocyclohexane) platinum (II)-loaded polymeric micelles directed to improved tumor targeting and enhanced antitumor activity. J Control Release. 2007;121(3):146-155.

21. Yamamoto Y, Hyodo I, Takigahira M, et al. Effect of combined treatment with the epirubicin-incorporating micelles (NC-6300) and 1,2-diaminocyclohexane platinum (II)-incorporating micelles (NC-4016) on a human gastric cancer model. Int J Cancer. 2014;135(1): 214-223.

22. Extra JM, Marty M, Brienza S, Misset JL. Pharmacokinetics and safety profile of oxaliplatin. Semin Oncol. 1998;25(2 Suppl 5):S13-S22.

23. Pasetto LM, D'Andrea MR, Rossi E, Monfardini S. Oxaliplatinrelated neurotoxicity: how and why? Crit Rev Oncol Hematol. 2006; 59(2):159-168.

24. Ling B, Authier N, Balayssac D, Eschalier A, Coudore F. Behavioral and pharmacological description of oxaliplatin-induced painful neuropathy in rat. Pain. 2007;128(3):225-234.

25. Sakurai M, Egashira N, Kawashiri T, Yano T, Ikesue H, Oishi R. Oxaliplatin-induced neuropathy in the rat: involvement of oxalate in cold hyperalgesia but not mechanical allodynia. Pain. 2009;147(1-3): $165-174$. 
26. Kawashiri T, Egashira N, Watanabe H, et al. Prevention of oxaliplatininduced mechanical allodynia and neurodegeneration by neurotropin in the rat model. Eur J Pain. 2011;15(4):344-350.

27. Norcini M, Vivoli E, Galeotti N, Bianchi E, Bartolini A, Ghelardini C. Supraspinal role of protein kinase $\mathrm{C}$ in oxaliplatin-induced neuropathy in rat. Pain. 2009;146(1-2):141-147.

28. Gamelin L, Boisdron-Celle M, Delva R, et al. Prevention of oxaliplatinrelated neurotoxicity by calcium and magnesium infusions: a retrospective study of 161 patients receiving oxaliplatin combined with 5-Fluorouracil and leucovorin for advanced colorectal cancer. Clin Cancer Res. 2004;10(12 Pt 1):4055-4061.
29. Grolleau F, Gamelin L, Boisdron-Celle M, Lapied B, Pelhate M, Gamelin E. A possible explanation for a neurotoxic effect of the anticancer agent oxaliplatin on neuronal voltage-gated sodium channels. J Neurophysiol. 2001;85(5):2293-2297.

\section{Publish your work in this journal}

The International Journal of Nanomedicine is an international, peerreviewed journal focusing on the application of nanotechnology in diagnostics, therapeutics, and drug delivery systems throughout the biomedical field. This journal is indexed on PubMed Central, MedLine, CAS, SciSearch ${ }^{\circledR}$, Current Contents ${ }^{\circledR} /$ Clinical Medicine,
Journal Citation Reports/Science Edition, EMBase, Scopus and the Elsevier Bibliographic databases. The manuscript management system is completely online and includes a very quick and fair peer-review system, which is all easy to use. Visit http://www.dovepress.com/ testimonials.php to read real quotes from published authors.

Submit your manuscript here: http://www.dovepress.com/international-journal-of-nanomedicine-journal 